\title{
CARBAPENEM RESISTANCE IN ESCHERICHIA COLI AND KLEBSIELLA PNEUMONIAE AMONG INDIAN AND INTERNATIONAL PATIENTS IN NORTH INDIA
}

\author{
NAMITA JAGGI ${ }^{1 *}$, NiRUPAMA CHATTERJEE ${ }^{2}$, VYOMA SINGH ${ }^{1}$, \\ SANTOSH KUMAR GIRI ${ }^{2}$, PRIYAMBADA DWIVEDI ${ }^{2}$, RAJESH PANWAR ${ }^{1}$ and \\ AJENDRA PRATAP SHARMA ${ }^{2}$ \\ ${ }^{1}$ Laboratory Services, Artemis Hospitals, Gurugram, India \\ ${ }^{2}$ Research and Education, Artemis Hospitals, Gurugram, India
}

(Received: 26 December 2018; accepted: 1 March 2019)

The aim of the study was to find out the carbapenem resistance rate and prevalence of different carbapenemase genes in Klebsiella pneumoniae and Escherichia coli from a North Indian corporate hospital that receives both Indian and international patients. A total of 528 clinical isolates of E. coli and K. pneumoniae were included in the study. All isolates that were found resistant to carbapenems by MIC testing (Vitek II Compact ${ }^{\circledR}$ ) were screened for NDM, OXA-48, VIM, and KPC genes by PCR. Sequencing of NDM gene and transmissibility by conjugation assay were checked on 22 randomly selected NDM-positive isolates. One hundred and fifty-six isolates $(29.54 \%)$ were carbapenem-resistant. The rate of carbapenem resistance was significantly higher in $K$. pneumoniae as compared to $E$. coli $(53.9 \%$ vs. $15.6 \% ; p<0.05)$. The NDM gene was found in $34.6 \%(54 / 156)$, OXA-48 in $31.4 \%(49 / 156)$, co-expression of NDM + OXA-48 in $15.3 \%(24 / 156)$ of the carbapenem-resistant isolates. VIM and KPC were absent in all isolates. NDM gene was significantly more prevalent in E. coli than $K$. pneumoniae $(p<0.05)$. All the tested isolates formed transconjugants and NDM-5 was the most common variant in both species $(15 / 22)$. The presence of plasmid-based NDM calls for stricter surveillance measures in our hospital settings.

Keywords: carbapenemase, NDM, OXA-48, Escherichia coli, Klebsiella pneumoniae

*Corresponding author; E-mail: namita@artemishospitals.com 


\section{Introduction}

Carbapenems are the antibiotics of choice for infections caused by cephalosporin-resistant bacteria. [1, 2]. However, resistance to carbapenems has currently become predominant in almost all groups of pathogenic bacteria including Enterobacteriaceae [2]. Carbapenem-resistant (CR) Enterobacteriaceae poses a serious threat to antibiotic therapy as it results in high rate of morbidity and mortality [3, 4]. Carbapenemases are the major mechanisms of resistance, and carbapenemase harboring isolates have been reported from across the globe with varying rates of prevalence. Globally, Klebsiella pneumoniae carbapenemase (KPC) is the most common carbapenemase, while in the Indian subcontinent, New Delhi metallo- $\beta$-lactamase (NDM), which supposedly originated in India in 2008 , is the most commonly reported carbapenemase [2, 5]. Various variants of NDM differing from each other in one or few nucleotides have emerged over the years [6, 7]. Besides NDM, oxacillinase-48 (OXA-48)-like enzymes have also been reported from India [8]. In India, NDM has spread to community and has been reported from public tap water and sewage isolates [9].

Escherichia coli and Klebsiella pneumoniae are the most common Gramnegative pathogens in clinical microbiology laboratories, and the resistance rate to carbapenems in these organisms is high in India [10]. The rise in antimicrobial resistance poses a serious challenge in centers where international patients come for treatment. This study was undertaken in a corporate hospital in North India that caters to both Indian and international patients with the aim to find out the prevalence of important carbapenemases in E. coli and K. pneumoniae.

\section{Materials and Methods}

Study design, bacterial isolates, and patient profile

The study was carried out at a tertiary care referral hospital in Gurugram, North India. The study design was approved by the institutional ethics committee. A total of 528 non-duplicated, consecutive E. coli and K. pneumoniae isolates were recovered from various clinical samples, such as urine, sputum, blood, pus, stool, tracheal aspirate, and body fluids. The samples were received in the clinical microbiology department from both inpatient [including intensive care unit (ICU)] and outpatient departments between November 2017 and February 2018. The details pertaining to patient's background, such as age, gender, nationality, locations, and mortality, were retrieved from the Hospital Information System. 
Antimicrobial susceptibility

Standard bacteriological techniques were used for the isolation of the organisms [11]. Identification of isolates and antibiotic susceptibility testing was performed on automated Vitek-2 compact system (Biomerieux, France). Isolates confirmed as E. coli and K. pneumoniae and showing resistance to carbapenems based on minimum inhibitory concentration (MIC) values (as determined by Vitek system and interpreted according to CLSI guidelines) [12] were further subjected to polymerase chain reaction (PCR) for determining the presence of various carbapenemase genes.

\section{Detection of carbapenemases by PCR and NDM sequencing}

Total DNA was extracted by the boiling lysis method as described earlier [13]. Detection of carbapenemase genes, such as NDM, OXA-48, Verona integron-encoded metallo- $\beta$-lactamase (VIM), and KPC, was carried out using PCR. The positive controls for the carbapenemase genes were obtained from the Microbiology Lab of Christian Medical College, Vellore, India. The PCR reaction was carried out in a 20- $\mu 1$ reaction consisting of $10 \mu \mathrm{l}$ of commercial $2 \times$ taq polymerase master mix (Qiagen cat\# 201443), $0.5 \mu \mathrm{M}$ of forward and reverse primers, and $2 \mu 1$ of DNA template. The PCR included initial denaturation of $95^{\circ} \mathrm{C}$ for $5 \mathrm{~min}$ followed by 35 cycles of denaturation at $95^{\circ} \mathrm{C}$ at $1 \mathrm{~min}$, annealing at $55^{\circ} \mathrm{C}$ for $1 \mathrm{~min}$ (for NDM annealing at $60^{\circ} \mathrm{C}$ ), extension at $72{ }^{\circ} \mathrm{C}$ for $1 \mathrm{~min}$, and a single cycle of final extension at $72^{\circ} \mathrm{C}$ for $7 \mathrm{~min}$. The amplified products were run on $1.5 \%$ agarose gel and visualized under UV light using a Gel Doc (Molecular Imager, Biorad, Hercules, CA, USA). The primer sequence for NDM, VIM, and KPC was taken from existing literature [14, 15]. Primer sequence for screening of OXA-48 (forward: GTTCATCCTTAACCACGCCC; reverse: CGGTAGCAAAGGAATGGCAA) and NDM sequencing (forward: ATTAGCCGCTGCATTGAT; reverse: GGGCCGTATGAGTGATTG) was designed during this study using Primer3 software (https://www.ncbi.nlm.nih. gov/tools/primer-blast/).

The identification of NDM variants was performed as follows: a portion of NDM gene spanning $743 \mathrm{bp}$ was amplified by PCR and amplicons were purified using a PCR purification kit (Qiagen cat\# 28004) and were sequenced (Applied Biosystems, ABI3730 XL, Foster City, CA, USA). The sequenced data were compared with sequences of various NDM determinants obtained from NCBI using BLAST software (http://www.ncbi.nlm.nhi.gov/blast/). 


\section{Conjugation}

Conjugation assay was performed by plate mating. Overnight cultures of donor strain and recipient strain of E. coli J53 $\left(\right.$ Azide $\left.^{\mathrm{R}}\right)$ were diluted 1:10 and 1:100, respectively, and allowed to grow at $37{ }^{\circ} \mathrm{C}$ for an hour. Conjugation by plate mating was performed by mixing these cultures in 1:1 ratio and streaking on Mueller-Hinton agar (MHA) plates followed by incubation at $37{ }^{\circ} \mathrm{C}$ for $4 \mathrm{~h}$. The transconjugants were selected by plating on MHA plates containing $2 \mu \mathrm{g} / \mathrm{ml}$ meropenem and $200 \mathrm{mg} / \mathrm{L}$ sodium azide. All transconjugants were tested for the presence of NDM by PCR.

\section{Statistical analysis}

The data are represented as absolute numbers and percentages. Nominal categorical data between the groups were compared by $\chi^{2}$ test or Fisher's exact test as appropriate. All major data analyses were performed using Microsoft Excel. For all statistical tests, a $p$ value less than 0.05 was taken to indicate a significant difference.

\section{Results}

A total of 528 isolates of $K$. pneumoniae and E. coli from 488 patients were included in this study. About $51.8 \%(n=253)$ of the patients were males and the median age of the patients was 55 years. The rate of carbapenem resistance was significantly higher in males as compared to females $(p<0.001)$. There was no significant difference in the carbapenem resistance rate between Indian and international patients $(p>0.05)$. Carbapenem resistance rate was significantly higher in the inpatients than outpatients $(p<0.0001)$. In addition, we observed gender- and location-wise differences in the distribution of the two organisms under the study. CR $K$. pneumoniae was significantly higher in males as compared to females $(p<0.05)$, but the same was not true in case of CR E. coli. The rate of carbapenem resistance according to gender, nationality, and location in all patients and in the two organisms is detailed in Table I.

Of the total 528 isolates, $156(29.54 \%)$ showed resistance to carbapenems. E. coli comprised $64.01 \%(338 / 528)$ of the total isolates; however, the rate of carbapenem resistance in $E$. coli was significantly less than $K$. pneumoniae $(15.6 \%$ vs. $53.9 \%$; $p<0.0001)$. K. pneumoniae dominated most sample types except for stool where E. coli was predominant. The majority (43.6\%) of 
Table I. Demographics of carbapenem-resistant patients

\begin{tabular}{|c|c|c|c|c|}
\hline \multirow{2}{*}{\multicolumn{2}{|c|}{ Total number of patients (488) }} & \multirow{3}{*}{$\begin{array}{c}\text { Total } \\
\text { carbapenem- } \\
\text { resistant }\end{array}$} & \multirow{3}{*}{$\frac{\text { Carbapenem-resistant }}{\text { K. pneumoniae }}$} & \multirow{3}{*}{$\begin{array}{c}\text { Carbapenem-resistant } \\
\qquad \text { E. coli } \\
27\end{array}$} \\
\hline & & & & \\
\hline \multirow[t]{2}{*}{ Gender } & Males (253) & & & \\
\hline & Females (235) & 48 & 27 & 21 \\
\hline \multirow[t]{2}{*}{ Nationality } & Indian (426) & 118 & 76 & 42 \\
\hline & International (62) & 18 & 12 & 6 \\
\hline \multirow[t]{3}{*}{ Location } & Outpatients (217) & 33 & 20 & 13 \\
\hline & Wards (152) & 53 & 32 & 21 \\
\hline & ICU (119) & 50 & 37 & 13 \\
\hline
\end{tabular}

Note: ICU: intensive care unit.

CR $K$. pneumoniae isolates was from ICU, whereas the major percentage of CR $E$. coli (47.1\%) was from wards. The distribution of CR E. coli and K. pneumoniae in various sample types and locations is depicted Figure 1.

The prevalence of NDM and OXA-48 in the overall isolates was $34.6 \%$ and $31.4 \%$, respectively. In $15.3 \%$ isolates, co-expression of NDM and OXA-48 was observed, while VIM and KPC were absent in all isolates. In $18.5 \%$ isolates, none of the tested carbapenemase genes were detected. The prevalence of

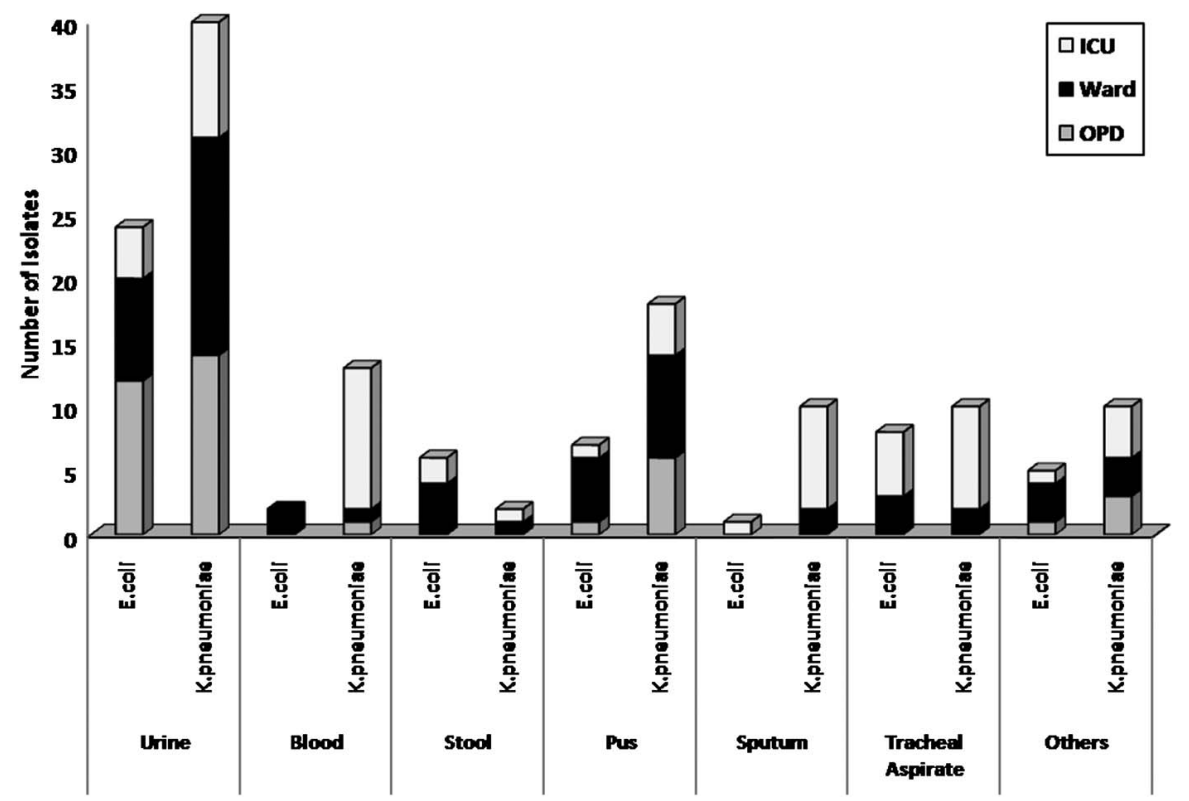

Figure 1. Distribution of $K$. pneumoniae and E. coli in different sample types and location 
carbapenemase genes NDM and OXA-48 in the two species and in various sample types was not uniform. NDM gene alone or in combination with OXA-48 was present in $77 \%$ of CR E. coli as compared to $35.9 \%$ of CR K. pneumoniae. The distribution of carbapenemases NDM and OXA-48 in the two organisms and various sample types is shown in Figure 2.

Twenty-two NDM-positive isolates were randomly selected for sequencing. NDM-5 was the predominant variant in both the species. Out of the 13 NDMpositive isolates of $K$. pneumoniae that were sequenced for the NDM gene, 9 were NDM-5 and 4 were NDM-1. In E. coli, 6 out of 9 were NDM-5 and one each was NDM-1, NDM-6, and NDM-7. All the derived sequences were submitted to GenBank and have been assigned the following accession numbers (MH683602MH683610 and MH-744357-MH744369). Conjugation assay was carried out to check the location (plasmid/chromosomal) on all the isolates that were sequenced. All the isolates formed transconjugants in conjugation assay that were positive for NDM in PCR, indicating the presence of NDM gene on plasmids.

In this study, 62 isolates came from international patients hailing from 16 different countries, mainly from West Asian, Eastern European, and African nations. Twenty-three isolates, those belonging to countries of Iraq, Afghanistan, Uganda, Kenya, Uzbekistan, Sudan, and UK, exhibited carbapenem resistance. Maximum numbers of isolates (36) were from Iraq, of which 13 showed carbapenem resistance. Of the $23 \mathrm{CR}$ isolates, 6 expressed the NDM gene of which 3 were from Iraqi nationals, and one each was from patients belonging to

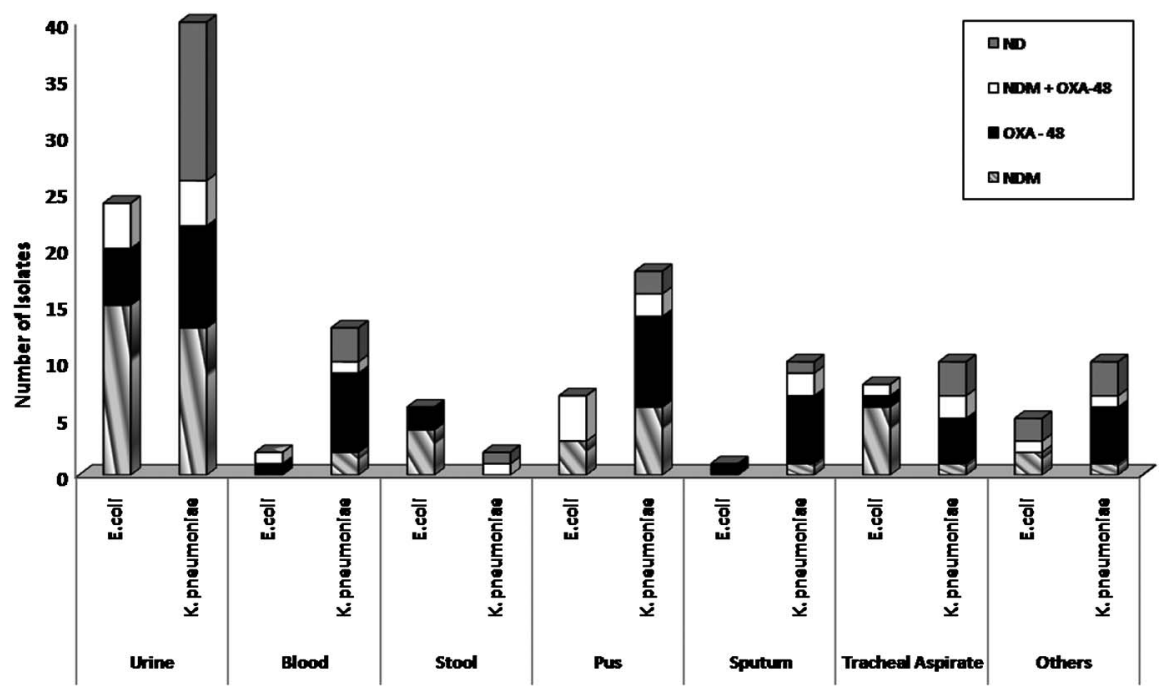

Figure 2. Distribution of carbapenemase in E. coli and K. pneumoniae and various sample types 
Uganda, Uzbekistan, and Sudan. OXA-48 expression was seen in seven isolates in patients belonging to Iraq (5), Afghanistan (1), and UK (1). Three isolates from Iraqi patients and two from Kenyan patients co-expressed the NDM and OXA-48 genes. In the remaining five isolates, none of the tested carbapenemases were detected. The isoforms of NDM observed in Iraqi patients were NDM-5, NDM-1, and NDM-6. NDM-5 was also found in one patient each from Uganda and Sudan.

As compared to OXA-48, NDM-carrying E. coli isolates were more likely to have a high MIC range for meropenem taking an arbitrary cut-off of $8 \mu \mathrm{g} / \mathrm{ml}$, while in $K$. pneumoniae a similar trend was seen for imipenem $(p<0.05)$. Isolates co-expressing NDM and OXA-48 were likely to have a higher MIC range than OXA-48 alone but the results were not statistically significant. There was no difference in the MIC range of the two carbapenems in the various variants of NDM.

Mortality rates were significantly higher in patients harboring CR isolates than the carbapenem-sensitive ones ( $21 \%$ vs. $7 \%$; $p<0.05)$. Within the CR group, the presence of carbapenemase genes did not have any significant effect on mortality rates $(p>0.05)$.

\section{Discussion}

Antibiotic resistance is a public health menace in India and the rate of carbapenem resistance is also showing an alarmingly increasing trend [16]. Although reports on CR bacteria and prevalence of carbapenemase genes from North India are frequent, we conducted this study in our hospital settings because it is a multispecialty set up and around $15 \%$ of our patients are from abroad. The study also will help us in modifying and upgrading existing antibiotic stewardship policies of the institution.

The rate of carbapenem resistance was significantly higher in males than in females and this was particularly true for CR K. pneumoniae. This could be due to the fact that majority of the CR K. pneumoniae was isolated from ICU, where $67 \%$ of the patients were males. Second, we did not find any remarkable difference in rate of carbapenem resistance in Indian and international patients. This was not surprising as the majority (56\%) of the foreign patients in the study was from Iraq where the rate of carbapenem resistance is high and the presence of NDM and OXA-48 genes in CR isolates has been previously reported [17, 18]. Isolates from foreign patients other than Iraq were represented by less than 10 samples.

Of the four carbapenemases screened in the study, NDM was the most common followed by OXA-48, which is similar to the previous findings from North India in the recent past [8]. However, distribution of the two genes in the two species under the study and in various sample types was not uniform. The rate 
of carbapenem resistance is higher in $K$. pneumoniae than in E. coli, which is in accordance with earlier reports [19]. The prevalence of NDM in E. coli was much higher than in $K$. pneumoniae. E. coli was abundant in stool and urine samples where the origin of infection is mostly from the gut microflora, suggesting that NDM-harboring E. coli might be present in the gut microflora in the community. On the other hand, blood and sputum samples were mainly reported from ICU and they showed a predominance of OXA-48-containing $K$. pneumoniae.

Various variants of NDM have been reported from North India and Delhi previously [20,21], but our data assume significance as it has been carried out on both Indian and international patients. This study identified NDM-5 as the most common variant in our hospital settings followed by NDM-1, which is probably the first in its kind of report. NDM-5 was isolated from samples of both Indian and international patients. Due to limitations, we are unable to ascertain the clonal relationship between these isolates. Moreover, the result of conjugation assay indicates plasmid-based location in all the isolates that were sequenced. This calls for stricter surveillance and infection control measures as plasmid-based genes can be transferred between species and spread rapidly.

OXA-48 is a weak carbapenemase that has the chance to be missed in phenotypic tests. However, in combination with carbapenem resistance mechanisms, such as efflux pump, porins, or other carbapenamse genes, OXA-48 increases the potency of carbapenem resistance manifolds [2]. Our results also support the above observations. In this study, the MIC values for imipenem were significantly high in $K$. pneumoniae isolates harboring NDM than those harboring OXA-48 and the similar was true for meropenem in E. coli isolates. Similarly, OXA-48 in combination with NDM had higher range of MIC for imipenem in $K$. pneumoniae, although the values were not statistically significant.

In this study, 27 isolates of $K$. pneumoniae and two isolates of $E$. coli were negative for all the tested carbapenemases. This indicates that these isolates of $K$. pneumoniae have mechanisms other than carbapenemase-like efflux pumps or porins to resist the carbapenems, which need further investigation. This baseline data generated from this study in a multispeciality hospital is important in view of the growing antibiotic resistance and flourishing medical tourism.

\section{Abbreviations}

NDM : New Delhi metallo- $\beta$-lactamase

OXA-48 : oxacillinase-48

VIM : Verona integron-encoded metallo- $\beta$-lactamase

KPC : Klebsiella pneumoniae carbapenemase 
PCR : polymerase chain reaction

MIC : minimum inhibitory concentration

CR : carbapenem-resistant

ND : none of the tested carbapenemase detected

\section{Acknowledgements}

The authors would like to thank Prof. Balaji Veeraraghavan, Christian Medical College, Vellore, for providing the positive control strains of $\mathrm{c}$ arbapenemase genes and George A. Jacoby, Lahey Clinic, Burlington, Massachusetts and Sandeep Ghatak. Division of Animal Health, ICAR Research Complex for NEH Region, Umiam, Meghalaya, India, for providing the E.coli J53 strain.

\section{Conflict of Interest}

None.

\section{References}

1. Jacoby, G. A., Munoz-Price, L. S.: The new beta-lactamases. N Engl J Med 352, 380-391 (2005).

2. van Duin, D., Doi, Y.: The global epidemiology of carbapenemase-producing Enterobacteriaceae. Virulence 8, 460-469 (2017).

3. Gupta, N., Limbago, B. M., Patel, J. B., Kallen, A. J.: Carbapenem-resistant Enterobacteriaceae: Epidemiology and prevention. Clin Infect Dis 53, 60-67 (2011).

4. Falagas, M. E., Tansarli, G. S., Karageorgopoulos, D. E., Vardakas, K. Z.: Deaths attributable to carbapenem-resistant Enterobacteriaceae infections. Emerg Infect Dis 20, 1170-1175 (2014).

5. Yong, D., Toleman, M. A., Giske, C. G., Cho, H. S., Sundman, K., Lee, K., Walsh, T. R.: Characterization of a new metallo-lactamase gene, bla $a_{\mathrm{NDM}-1}$, and a novel erythromycinesterase gene carried on a unique genetic structure in Klebsiella pneumoniae sequence type 14 from India. Antimicrob Agents Chemother 3, 5046-54 (2009).

6. Shrestha, B., Tada, T., Shimada, K., Shrestha, S., Ohara, H., Pokhrel, B. M.: Emergence of various NDM-type-metallo- $\beta$-lactamase-producing Escherichia coli clinical isolates in Nepal. Antimicrob Agents Chemother 61, 01425-17 (2017).

7. Rahman, M., Shukla, S.K., Prasad, K. N., Ovejero, C. M., Pati, B. K., Tripathi, A., Singh, A., Srivastava, A. K., Gonzalez-Zorn, B.: Prevalence and molecular characterisation of New Delhi metallo-lactamases NDM-1, NDM-5, NDM-6 and NDM-7 in multidrug-resistant Enterobacteriaceae from India. Int J Antimicrob Agents 44, 30-37 (2014). 
8. Mohanty, S., Gajanand, M., Gaind, R.: Identification of carbapenemase-mediated resistance among Enterobacteriaceae bloodstream isolates: A molecular study from India. Indian J Med Microbiol 35, 421-425 (2017).

9. Walsh, T. R., Weeks, J., Livermore, D. M., Toleman, M. A.: Dissemination of NDM-1 positive bacteria in the New Delhi environment and its implications for human health: An environmental point prevalence study. Lancet Infect Dis 11, 355-62 (2011).

10. Veeraraghavan, B., Jesudason, M. R., Prakasah, J. A. J., Anandan, S., Sahni, R. D., Pragasam, A. K., Bakthavatchalam, Y. D., Selvakumar, R. J., Dhole, T. N., Rodrigues, C., Roy, I., Joshi, I. S., Chaudhuri, B. N., Chitnis, D. S.: Antimicrobial susceptibility profiles of gram-negative bacteria causing infections collected across India during 2014-2016: Study for monitoring antimicrobial resistance trend report. Indian J Med Microbiol 36, 32-36 (2018).

11. Collee, J. G., Miles, R. S., Wan, B.: Tests for the identification of bacteria. In Collee, J. G., Fraser, A. G., Marmion, B. P., Simmons, A. (eds): Mackie and McCartney Practical Medical Microbiology, $14^{\text {th }}$ Edition. Churchill Livingstone, Edinburgh, 1996, pp. 131-50.

12. Clinical and Laboratory standards Institute [CLSI]: Performance Standards for Antimicrobial Susceptibility Testing, 28th Informational Supplement M100. CLSI, Wayne, PA, 2006.

13. Dashti, A. A., Jadaon, M. M., Abdulsamad, A. M., Dashti, H. M.: Heat treatment of bacteria: A simple method of DNA extraction for molecular techniques. Kuwait Med J 41, 117-122 (2009).

14. Manchanda, V., Rai, S., Gupta, S., Rautela, R. S., Chopra, R., Rawat, D. S., Verma, V. N., Singh, N. P., Kaur, I. R., Bhalla, P.: Development of TaqMan real-time polymerase chain reaction for the detection of the newly emerging form of carbapenem resistance gene in clinical isolates of Escherichia coli, Klebsiella pneumoniae, and Acinetobacter baumannii. Indian J Med Microbiol 29, 249-53 (2011).

15. Dallenne, C., Da Costa, A., Decré, D., Favier, C., Guillaume, A.: Development of a set of multiplex PCR assays for the detection of genes encoding important $\beta$-lactamases in Enterobacteriaceae. J Antimicrob Chemother 65, 490-495 (2010).

16. Patel, I., Hussain, R., Khan, A., Ahmad, A., Khan, M. U., Hassalai, M. A. A.: Antimicrobial resistance in India. J Pharmaceut Policy Pract 10, 27 (2017).

17. Hussein, N. H.: Emergence of NDM-1 among carbapenem-resistant Klebsiella pneumoniae in Iraqi hospitals. Acta Microbiol Immunol Hung 65, 211-227 (2018).

18. Al-Charrakh, A. H., Al-Awadi, S. J., Mohammed, A. S.: Detection of metallo- $\beta$-lactamase producing Pseudomonas aeruginosa isolated from public and private hospitals in Baghdad, Iraq. Acta Medica Iranica 54, 107-113 (2016).

19. Sharma, A., Bakthavatchalam, Y. D., Gopi, R., Anandan, S., Verghese, V. P., Veeraraghavan, B.: Mechanisms of Carbapenem resistance in K. pneumoniae and E. coli from bloodstream infections in India. J Infect Dis Ther 4, 4 (2016).

20. Grover, S. S., Gupta, N., Gandhoke, I., Batra, J., Hans, C., Khare, S.: New Delhi metallo- $\beta$ lactamase-type carbapenemases producing Escherichia coli isolates from hospitalized patients: A pilot study. Indian J Med Res 146, 105-110 (2017).

21. Ahmad, N., Ali, S. M., Khan, A. U.: Detection of New Delhi metallo- $\beta$-lactamase variants NDM-4, NDM-5, and NDM-7 in Enterobacter aerogenes isolated from a neonatal intensive care unit of a North India hospital: A first report. Microb Drug Resist 24, 161-165 (2018). 